\title{
Giant peritoneal loose body: A case report
}

\author{
SHANSHAN GUO ${ }^{1,2 *}$ HAIJUAN YUAN ${ }^{1 *}$, YINGYING XU ${ }^{1,3^{*}}$, PING CHEN $^{1}$ and LIANG ZONG ${ }^{1,3}$ \\ ${ }^{1}$ Department of Gastrointestinal Surgery, Northern Jiangsu People's Hospital, Clinical Medical College, \\ Yangzhou University, Yangzhou, Jiangsu 225001; ${ }^{2}$ Department of Oncology, Graduate School of Medicine, Dalian Medical \\ University, Dalian, Liaoning 116044; ${ }^{3}$ Department of Gastrointestinal Surgery, Nanjing Drum Tower Hospital, \\ The Affiliated Hospital of Nanjing University Medical School, Jiangsu, Nanjing, 210008, P.R. China
}

Received January 8, 2019; Accepted February 15, 2019

DOI: $10.3892 /$ br.2019.1208

\begin{abstract}
Peritoneal loose body (PLB) is a rare finding and it is usually incidentally discovered during laparotomy, examination or autopsy, as it is usually asymptomatic or presents with non-specific symptoms. In particular, giant PLBs, measuring $>50 \mathrm{~mm}$ in maximum diameter, are even more rare, and only a few cases of laparoscopic extraction of these giant bodies have been reported in the literature to date. We herein describe our experience with the diagnosis and treatment of a giant PLB in a 49-year-old male patient who was admitted with complaints of intermittent pain the in lower abdomen 3 months earlier. An abdominal computed tomography scan revealed a $5.5 \times 5.0-\mathrm{cm}$ midline mass with central high density. The diagnosis was confirmed by open surgery and postoperative pathological examination. The postoperative recovery of the patient was uneventful and he was discharged from the hospital 7 days after surgery.
\end{abstract}

\section{Introduction}

Peritoneal loose bodies (PLBs) are quite rare and usually present as small, white or pale gray, egg-shaped objects with a smooth glistening surface. They are usually located freely in the peritoneal cavity (1) and rarely cause symptoms. PLBs are usually 5-20 $\mathrm{mm}$ in diameter and are diagnosed via laparotomy or autopsy by accident (2). Matsubara et al (3) reviewed 20 reported PLB cases and determined that PLB is more

Correspondence to: Professor Liang Zong, Department of Gastrointestinal Surgery, Northern Jiangsu People's Hospital, Clinical Medical College, Yangzhou University, 98 Nantong West Road, Yangzhou, Jiangsu 225001, P.R. China

E-mail: 250537471@qq.com

*Contributed equally

Abbreviations: PLB, peritoneal loose body; CT, computed tomography; MRI, magnetic resonance imaging

Key words: peritoneal loose body, appendices epiploicae, case report common in males, with a male:female ratio of 17:3. Although the pathogenesis of PLB remains unclear, it is widely accepted that they arise from infarcted appendices epiploicae, which then go through several sequential processes, including saponification, calcification and fibrosis (4). PLBs then increase in size by accumulating albumin from exudative peritoneal fluid (4). In the pathological examination of PBLs, hyperplastic fibrillar collagen with partial microcalcifications are usually used as criteria for diagnosis $(5,6)$. In most cases, patients present with non-specific symptoms and do not require treatment. However, in cases of giant PLBs, surgical intervention is important for treatment, as giant PLBs are more likely to cause acute or life-threatening symptoms (7). Furthermore, other diseases may not be fully excluded following radiological imaging alone so surgical exploration may be necessary for the definitive diagnosis and treatment of PLBs (8).

\section{Case report}

A 49-year-old man was admitted to Northern Jiangsu People's Hospital in March 2016 with a complaint of intermittent pain in the lower abdomen 3 months earlier. The patient had no fever, nausea or vomiting. The physical examination was unremarkable. The medical history was significant for acute appendicitis $\sim 4$ years earlier. At that time, only conservative treatment with antibiotics was administered instead of an appendectomy.

The laboratory tests revealed high levels of $\gamma$-glutamyltranspeptidase (80 U/1; normal range, 0-50 U/1), direct bilirubin and alanine aminotransferase, and increased levels of carcinoembryonic antigen (CEA); infectious markers included positive hepatitis B virus (HBV) markers, namely $\mathrm{HB}$ surface antigen, anti-HBe antibody and HB core antibody. An abdominal computed tomography $(\mathrm{CT})$ scan revealed a $5.5 \times 5.0-\mathrm{cm}$ midline mass with central high density (Fig. 1). In addition, the CT examination revealed a fatty liver. Abdominal open surgery was performed to remove the mass, and the postoperative pathological examination revealed that it was composed of hyperplastic fibrillar collagen with partial microcalcifications.

The postoperative recovery of the patient was uneventful and he was discharged from the hospital 7 days after surgery. The intermittent pain in the lower abdomen resolved immediately after removal of the mass. After a follow-up period of two years, the patient had no abnormal complaints. 

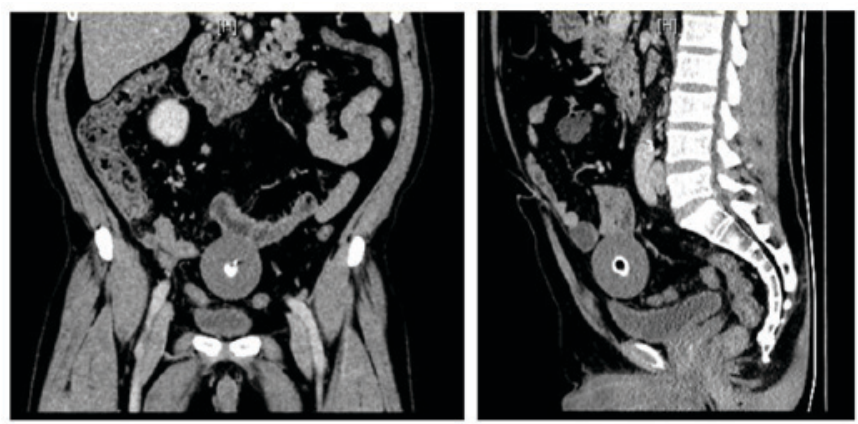

Figure 1. Anteroposterior (left) and lateral (right) computed tomography images of the patient.

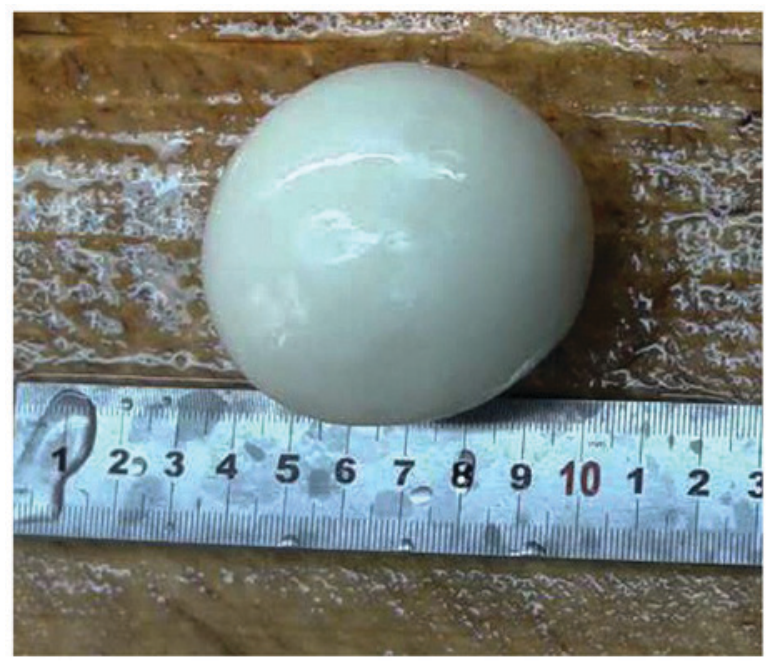

Figure 2. Macroscopic appearance of the giant peritoneal loose body.

\section{Discussion}

PLBs are a rare occurrence, and only a few cases of giant PLBs $(>5 \mathrm{~cm}$ in diameter) have been reported in the literature to date. PLBs are usually incidentally discovered during surgery or examination. The majority of PLBs are asymptomatic, but a small proportion of giant PLBs may cause symptoms, such as abdominal pain, bowel obstruction, urinary retention or urinary frequency (9). Matsubara et al reviewed 20 cases of giant PLBs reported in the literature and reported that PLBs were more common in men, with a male:female incidence of 17:3 (3). The majority of the patients were aged $>50$ years. In addition, most cases shared a distinct histological characteristic, namely calcified necrosis of fat tissue with hypocellular fibrolamellar tissue and numerous microcalcifications.

Clinically, PLBs must be differentiated from other tumors, such as mesenteric tumors, leiomyoma and teratoma. Abdominal CT examination may be helpful, as it demonstrates the characteristic features of PLBs, which are well-defined oval or round soft tissue masses with central calcification, usually located in the abdomen, with a distinct fat plane around the mass separating it from adjacent organs (10). Since PLBs are freely mobile, additional scanning in the prone position or a follow-up CT examination can demonstrate the change in location. Moreover, on magnetic resonance imaging (MRI) examination, PLB appears as a well-defined, low-intensity mass on both T1- and T2-weighted images (11). The MRI signal is similar to that of muscle, and a central high-intensity area may be seen on T1-weighted images. PLBs do not exhibit any enhancement, which is useful in differential diagnosis, as leiomyoma and teratoma exhibit contrast enhancement. Performing CT and MRI is crucial for identifying the characteristic features of PLB and establishing an accurate diagnosis.

The pathogenesis of PLBs remains unclear; however, the general consensus is that PLBs are derived from the epiploic appendices via sequential torsion, infarction, saponification and calcification. Sussman and Murdock described a rare case of a PLB in a 62-year-old patient in 2015 (9). As Sussman and Murdock (9) mentioned, the formation of the PLB was attributed to the torsion and detachment of an epiploic appendage, with subsequent transformation into a fibrotic mass. With continuous peritoneal exudate deposition on the surface, PLBs may slowly grow to a larger size. However, although there are a number of cases with severe abdominal or pelvic inflammation, only few result in the formation of a PLB, suggesting that some specific condition may be required. In the present case, CT imaging revealed a fatty liver, and the laboratory tests revealed a compromised liver function and increased CEA levels. It may be hypothesized that the compromised liver function may have increased the volume of peritoneal exudate, thereby facilitating PLB formation.

Of note, the formation of the PLB resembles that of a pearl, which originates from an external stimulus, such as a parasite, inducing a defense mechanism in mollusks, which then create a pearl sac to seal it off (Fig. 2).

\section{Acknowledgements}

Not applicable.

\section{Funding}

The present study was supported by the Jiangsu Natural Science Foundation (grant no. BK20180274).

\section{Availability of data and materials}

All the information relevant to the present study are available from the corresponding author on reasonable request.

\section{Authors' contributions}

SG drafted the manuscript and examined the patient; PC assisted in the preparation of the manuscript and examined the patient; LZ critically reviewed the manuscript and examined the patient. All authors have read and approved the final version of this manuscript.

\section{Ethics approval and consent to participate}

Not applicable.

\section{Patient consent for publication}

Written informed consent was obtained from the patient for the publication of the case details and any associated images. 


\section{Competing interests}

The authors declare that they have no competing interests to disclose.

\section{References}

1. Takabe K, Greenberg JI and Blair SL: Giant peritoneal loose bodies. J Gastrointest Surg 10: 465-468, 2006.

2. Zhang H, Ling YZ, Cui MM, Xia ZX, Feng Y and Chen CS: Giant peritoneal loose body in the pelvic cavity confirmed by laparoscopic exploration: A case report and review of the literature. World J Surg Oncol 13: 118, 2015.

3. Matsubara K, Takakura Y, Urushihara T, Nishisaka T and Itamoto T: Laparoscopic extraction of a giant peritoneal loose body: Case report and review of literature. Int J Surg Case Rep 39: 188-191, 2017.

4. Sewkani A, Jain A, Maudar K and Varshney S: 'Boiled egg' in the peritoneal cavity-a giant peritoneal loose body in a 64-year-old man: A case report. J Med Case Reports 5: 297, 2011.
5. Asabe K, Maekawa T, Yamashita Y and Shirakusa T: Endoscopic extraction of a peritoneal loose body: A case report of an infant. Pediatr Surg Int 21: 388-389, 2005.

6. Van Zyl C, Davis R, Hurter D and Van Der Westhuizen G: Giant peritoneal loose bodies. S Afr J Rad. 19: 730-733, 2015.

7. Elsner A, Walensi M, Fuenfschilling M, Rosenberg R and Mechera R: Symptomatic giant peritoneal loose body in the pelvic cavity: A case report. Int J Surg Case Rep 21: 32-35, 2016.

8. Obaid M and Gehani S: Deciding to Remove or Leave a Peritoneal Loose Body: A Case Report and Review of Literature. Am J Case Rep 19: 854-857, 2018.

9. Sussman R and Murdock J: Images in clinical medicine. Peritoneal loose body. N Engl J Med 372: 1359, 2015.

10. Gayer $\mathrm{G}$ and Petrovitch I: CT diagnosis of a large peritoneal loose body: A case report and review of the literature. Br J Radiol 84: e83-e85, 2011.

11. Takayama S, Sakamoto M and Takeyama H: Clinical challenges and images in GI. Image 1: huge peritoneal loose body in the pelvic cavity. Gastroenterology 136: 404, 730, 2009. 